\title{
Perceptual learning in human and nonhuman animals: A search for common ground
}

\author{
GeOFHey HaLL \\ University of York, York, England
}

\begin{abstract}
Perceptual learning has been extensively studied in both human and nonhuman animals, but the two lines of research have, for the most part, developed independently, addressing seemingly rather different issues by rather different methods. It has been argued, however, that analysis of the disparate phenomena studied in experiments on perceptual learning reveals that in all the studies, the essential feature is that appropriate training allows behavior to come to be controlled by the unique features, rather than by the common features, of similar stimuli. It has further been argued that experiments with nonhuman animals have established the existence of a range of learning processes that allow this to occur, and that these processes have general relevance, applying to humans as well as to animals.
\end{abstract}

In his introductory chapter for a book (Fahle \& Poggio, 2002) on perceptual learning, Fahle attempted to specify the nature of his topic by drawing a distinction between this and other forms of learning. This attempt produced a list of things that perceptual learning is not. Unlike other forms of learning, perceptual learning is said not to be based on "cognitive changes;" it is "relatively independent from conscious experience;" it does not involve declarative memory (after it has occurred, people cannot say what they are doing differently); and "it differs from associative learning because it relies neither on the mechanisms of classical nor on instrumental conditioning" (p. ix). The neuronal basis of the learning is held to occur early in processing (e.g., in the sensory cortex) and does not require the involvement of other structures (such as the hippocampus) that are said to be necessary for the consolidation of other types of learning.

It is perhaps significant that almost the whole of the Fahle and Poggio (2002) book concerns studies of perceptual learning done with human subjects, and that it pays only passing acknowledgement to the body of work (see, e.g., Hall, 1991) done with animal subjects. Perhaps just as significant is that the work reviewed in the book comes largely from scientists whose primary interest has been in perception and psychophysics and whose concept of learning is guided by the common-sense interpretation suggested by everyday experience. Equally, those working with animal subjects have had a primary interest in learning and have had little first-hand knowledge of modern work on perception, tending to treat it as a subsidiary process supplying the raw material for their learning mechanisms. However this may be, the negative aspects of the definitions offered by Fahle do little to foster a meeting of minds. Animal learning theorists often have a profound attachment to associative explanations, have shown little interest in the anatomical substrate of the changes they study, and (obviously) have shown little regard to whether or not the changes in behavior they observe are dependent on conscious experience.

These introductory comments may seem to bode ill for the prospect of establishing a fruitful dialogue between students of human and of animal perceptual learning, but, in fact, I remain optimistic on this matter. The divergences just noted stem from the attempt to define perceptual learning in negative terms. I want to argue that these divergences are not fundamental, and that if we concentrate on what perceptual learning is, rather than is not, we will be able to discern a set of features that are common to both approaches to the topic. in what follows, I hope to demonstrate that these approaches have more in common than might at first appear, and that concepts and experimental techniques from one area may be fruitfully applied to elucidate the whole. My first step, therefore, is to review (even if only briefly; for a fuller account, see Hall, 2008) the range of things that have been taken as instances of perceptual learning, with the intention of identifying the core features of the phenomenon.

\begin{abstract}
A Sampling of the Phenomena
Much work on perceptual learning in humans has been devoted to demonstrating how practice can improve performance on tasks designed to assess what may be termed simple sensory thresholds (although the very fact that they show such plasticity indicates that they are not all that simple). An experiment by Shiu and Pashler (1992) provides a convenient example of this. The participants were required to fixate a central point while, in the periphery, a pair of short $(1.5-\mathrm{cm})$ lines was presented briefly, one
\end{abstract}

G. Hall, g.hall@psych.york.ac.uk 

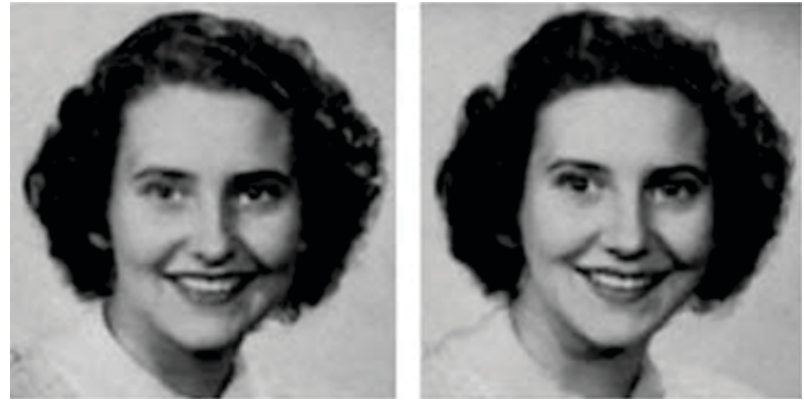

Figure 1. Two of the faces used in the study by Mundy, Honey, and Dwyer (2007). These were created by morphing between two already similar faces. The resulting pair (shown here) consists of one with $73 \%$ of Face 1 and $27 \%$ of Face 2, and the other with $73 \%$ of Face 2 and $23 \%$ of Face 1.

after another. The lines were oriented either $7^{\circ}$ or $10^{\circ}$ from the vertical, and the subjects were required to say whether the members of the pair were the same or different. The task was initially almost impossibly difficult, but after extensive training performance began to improve. When given feedback (when told that their responses were right or wrong), performance rose to about $70 \%$ correct after about 200 trials, although even the subjects who had been trained without feedback showed some modest improvement. Transfer studies helped to determine the nature of the effect; for example, the fact that performance fell back when the lines were presented in a different retinal location argues against the possibility that the effect might depend on some general learning process that allowed the subjects to become proficient with this particular testing procedure. Essentially similar results have been obtained for a variety of other simple discrimination tasks - for example, for auditory frequency (Demany, 1985), sinusoidal gratings (Fiorentini \& Berardi, 1980), the direction of movement of an array of moving dots (Ball \& Sekuler, 1982), the discrimination of visual texture (Karni \& Sagi, 1991), and so on.

In the popular imagination, however, perceptual learning tends to be equated with the performance shown by certain individuals on much more complex discriminations. I refer to the abilities of various experts: tea tasters, masters of wine, chicken sexers, skilled radiographers, and so on. How such experts acquire their abilities has not been the subject of much direct empirical investigation (but see, e.g., Biederman \& Shiffrar, 1987; MylesWorsley, Johnston, \& Simons, 1988), but the ability of ordinary mortals to improve their performance on complex discriminations has. Figure 1 shows an example of two of the stimuli used in a study of perceptual learning by Mundy, Honey, and Dwyer (2007). The subjects (undergraduate students) were told that one of these near-identical "twins" was left-handed and that one was right-handed, and that their task was to guess (feedback was given on this test) which was which. With the faces presented sequentially, this discrimination proved difficult to acquire. It was made easier, however, by giving the subjects preexposure to the stimuli-presenting the two faces repeatedly five times each before the test task was begun. No response was required during this preexposure phase (the subjects were simply told to count the number of times a face appeared on the screen), indicating that feedback is not required to produce a perceptual learning effect. Interestingly, it was found that the effect was obtained only when the two faces were presented in alternation during preexposure; pretraining in which all examples of each face were presented in separate blocks of trials was ineffective.

For an example of an experiment on perceptual learning in nonhuman animals, one can conveniently turn to a study by Symonds and Hall (1995). The experimental subjects were rats and the stimuli were different flavors, but the design and results constituted a close parallel to those reported by Mundy et al. (2007). The experiment is summarized in Table 1. There were three groups of subjects: One received no preexposure; one experienced alternating presentations of Flavors A and B; and one received equivalent exposure, with all presentations of A occurring as one block and all of B occurring as a separate block. In order to assess how these procedures influenced the ability of the rats to discriminate Flavor A from Flavor B, we made use of the flavor-aversion learning procedure. All rats received conditioning trials in which Flavor A was followed by a nausea-inducing injection, which was sufficient to establish an aversion to A. They were then given a test in which they were allowed to consume Flavor B. If the rats were to have difficulty in discriminating between Flavors A and B, the aversion established to A would generalize to B, and consumption would be suppressed. Such generalization is just what was observed for the rats that were given no preexposure or the blocked schedule (see Figure 2), but those in the intermixed condition consumed $\mathrm{B}$ readily, indicating that this form of preexposure allowed them to discriminate it from A. The reliability of this form of the perceptual learning effect has been established by many subsequent studies using the flavor-aversion procedure (e.g., Bennett \& Mackintosh, 1999; Blair \& Hall, 2003; Mondragón \& Hall, 2002), and its generality has been established in studies with other species (see, e.g., Honey, Bateson, \& Horn, 1994; Mundy, Dwyer, \& Honey, 2006).

\section{Analysis}

Although quite different in many respects, it is possible to see that the examples just cited have certain aspects in common. In all of them, the test task required the subject

Table 1

Design of Experiment by Symonds and Hall (1995)

\begin{tabular}{lccc}
\hline \multicolumn{1}{c}{ Group } & Preexposure & Conditioning & Test \\
\hline Control & - & $\mathrm{A}+$ & $\mathrm{B}$ \\
Intermixed & $\mathrm{A} / \mathrm{B} / \mathrm{A} / \mathrm{B} \ldots$ & $\mathrm{A}+$ & $\mathrm{B}$ \\
Blocked & $\mathrm{A}, \mathrm{A}, \ldots \mathrm{B}, \mathrm{B}$ & $\mathrm{A}+$ & $\mathrm{B}$ \\
\hline
\end{tabular}

Note-A and B represent different flavors; + represents an injection of lithium chloride. The control and intermixed groups experienced four presentations of each flavor during preexposure; there were three conditioning trials and a single test trial. 


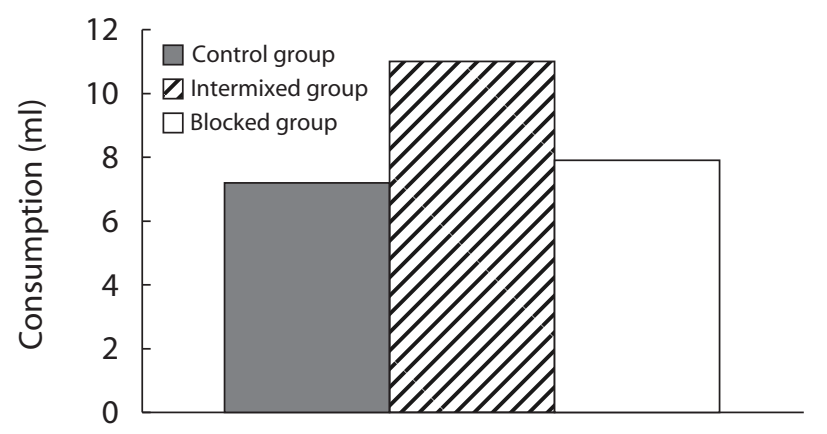

Figure 2. Results of the test phase (group mean consumption of Flavor B) in the experiment by Symonds and Hall (1995) (see Table 1). Rats in the intermixed and blocked groups were given preexposure to Flavors $A$ and $B$. The intermixed group received the flavors on alternate trials; the blocked group experienced the flavors on separate blocks of trials. The control group received no preexposure. After aversion conditioning with $A$, generalization to $B$ was tested.

to discriminate between two similar stimuli. To understand how experience can help with this discrimination, it will be useful for one to specify what the formation of a discrimination involves. The situation is depicted in Figure 3 . The two stimuli are represented by the overlapping circles $A$ and $B$. The overlap indicates that they have some features in common (marked $x$ in the figure): This is what makes them similar. They also have unique features (marked $a$ and $b$ in the figure). Success in discrimination requires that the subject's response come under the control of the unique features ( $a$ and $b$ ) rather than of the common features $(x)$ of the stimuli.

This perspective prompts a definition of perceptual learning: It may be regarded as "the learning process (or processes) that increases the effectiveness of the unique stimulus elements and/or reduces that of common stimulus elements, thus facilitating discrimination between similar stimuli" (Hall, 2008, p. 110). Note that this definition is neutral about the processes involved: They may involve association formation or they may not; they may involve declarative memory or they may not; they may involve cognitive changes or they may not. Which mechanisms are involved is a matter for empirical investigation; they do not form part of the definition. Thus, by accepting this definition, we can eliminate (or at least, bypass) the supposed distinctions that were alluded to in the introduction to the present article that create an apparent divide between animal and human perceptual learning. We may hope that empirical investigation - whether conducted with animals or with humans - will yield explanations of general applicability; that is, a learning mechanism that is capable of enhancing the effectiveness of a unique stimulus feature might be expected to operate generally, regardless of whether this feature is one that distinguishes one face from another (for people) or one flavor from another (for rats).

\section{Associative Processes and the Role of Feedback}

Many of the procedures described as examples of perceptual learning procedures can be construed as involving a simple discrimination learning task. The task is simple in that the rule describing it is simple, although the stimuli may be complex, difficult to discriminate, or both. The rule, however, is just that one stimulus is followed by one consequence, and the other by another. For experiments with people as the subjects, the consequence (feedback) is likely to be some cue telling them that their response is right or wrong; for rats, it may be the presence or absence of a reinforcer.

Standard theories of (animal) discrimination learning have no trouble in explaining improvement on such a task (it is what they were designed to do; see, e.g., Rescorla \& Wagner, 1972). Using the terminology outlined previously, the task reduces to training in which the ax compound (Stimulus A) is followed by Outcome 1 (O1), and the $b x$ compound (Stimulus B) by Outcome 2 (O2). Standard error-correction models (such as that proposed by Rescorla \& Wagner, 1972) readily predict that the common cues $(x)$ will be "neutralized" and that the $a$ cues will become strongly associated with $\mathrm{O} 1$, and the $b$ cues with $\mathrm{O} 2$. Discrimination (differential response to the two stimuli), which was absent in the beginning, will become well established by such training. This improvement in performance (which, in principle, could be all that is required to explain some examples of the abilities shown by experts) would not be perceptual learning as it was defined previously, in that it would not involve the changes in stimulus effectiveness that were central to my definition. This does not mean, however, that associative mechanisms play no part in perceptual learning, so defined. Next, I will consider three possible ways in which the associative processes engaged by discrimination training might also produce changes in either the effectiveness or the features of stimuli.

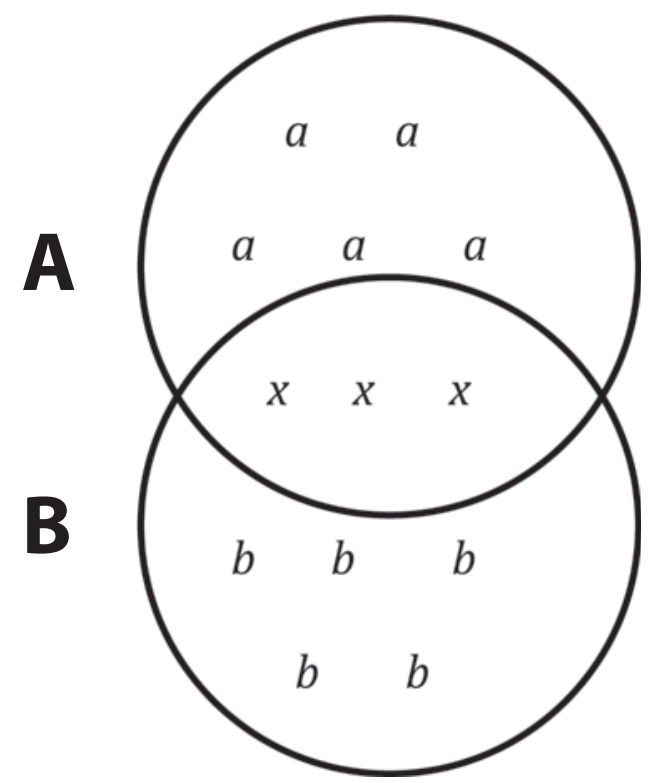

Figure 3. Each circle represents a stimulus (A or B) that is made up of a set of features (or elements). Some features are unique to a given stimulus (the $a$ elements for $A$; the $b$ elements for $B$ ); other features $(x)$ are held in common and thus fall into the area of overlap of $A$ and $B$. 


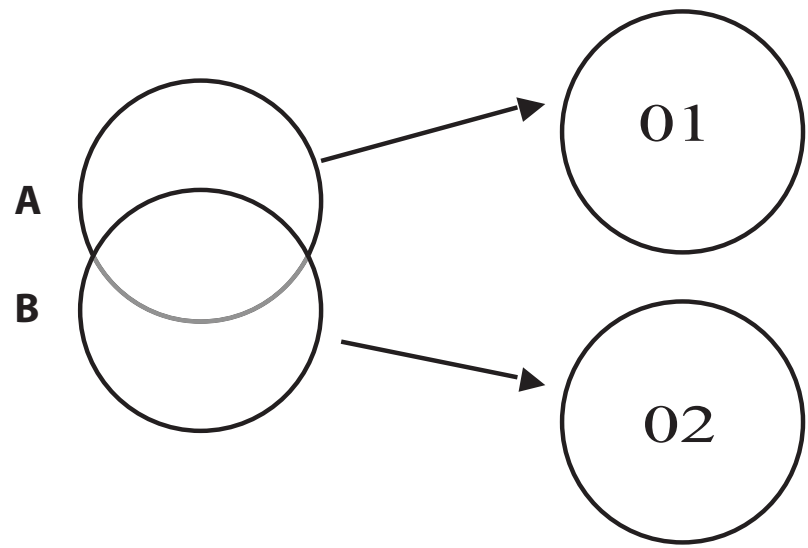

Figure 4. Associative structure established by discrimination training in acquired distinctiveness and acquired equivalence. The overlapping circles represent two similar stimuli, A and B (the area of overlap representing features held in common); arrows represent associative links. As a result of training, $A$ and $B$ have formed associations with quite different events (Outcome 1 and Outcome 2).

Acquired distinctiveness. The first possibility is summarized in Figure 4. The arrows represent the associations presumed to be formed when Stimulus A is reliably followed by $\mathrm{O} 1$ and Stimulus B by O2. The absence of overlap between the representations of $\mathrm{O} 1$ and $\mathrm{O} 2$ is meant to indicate that these are very different events. When such an associative structure has been established, the presentation of $\mathrm{A}$ alone will activate the representation of $\mathrm{O} 1$, and the presentation of $\mathrm{B}$ alone will activate the representation of O2. It has been repeatedly observed that establishing this associative structure facilitates further learning when a new discrimination between $\mathrm{A}$ and $\mathrm{B}$ is required. The classic instance of the effect is the case in which A and B are initially associated with different verbal labels prior to a test phase in which the subject must learn to make different motor responses to A and B. However, this effect has been demonstrated in many other training paradigms (see Hall, 1991, for a review). Importantly, this facilitation effect is found when the new task is quite different from that used in training. Positive transfer to the new task is assumed to depend, therefore, not on the specific associations formed in the first phase, but on some change in the properties of the cues; they are said to have acquired distinctiveness.

The standard explanation for this effect goes back as far as James (1890; but see Hall, Mitchell, Graham, \& Lavis, 2003, for a modern interpretation). The essential feature of the explanation is that further discrimination between A and B will be facilitated because the patterns of activation evoked by these events have been rendered more different as a consequence of the initial phase of discrimination training. In initial training, the discrimination required was between $a x(\mathrm{~A})$ and $b x(\mathrm{~B})$; however, in any further task involving these same stimuli, the discrimination is between $a x$ plus the associatively activated representation of $\mathrm{O} 1$, and $b x$ plus the associatively activated representation of $\mathrm{O} 2$. Since $\mathrm{O} 1$ and $\mathrm{O} 2$ have been chosen to be very different, subsequent performance will be facilitated. Because such an explanation involves a change in how stimuli that are relevant to solving the discrimination are represented, it is a mechanism consistent with the unifying definition of perceptual learning that was offered earlier.

Learned changes in associability. Mackintosh (1975) proposed a theory of attention in conditioning that supplies a further possible explanation for the acquired distinctiveness effect. He suggested that the readiness with which a given cue would enter into associations can be modified by experience. Specifically, he suggested that the associability of a cue that predicts an outcome more reliably than others will increase, and that of a cue that predicts the outcome less well than others will decline. Thus, training that established the structure shown in Figure 4 would increase the associability of the $a$ and $b$ components of the stimuli and reduce that of the $x$ components. A new task requiring a discrimination between $\mathrm{A}$ and $\mathrm{B}$ would thus be learned more readily.

Although intuitively appealing, direct evidence for the central assertion of this account - that predictive cues experience an increase in associability-has been hard to come by. Indeed, direct investigation using simple conditioning procedures has produced results suggesting that the opposite may be true (Hall \& Pearce, 1979; Pearce \& Hall, 1980). Recently, however, experiments using more complex discrimination procedures have generated encouraging results (see, e.g., Bonardi, Graham, Hall, \& Mitchell, 2005; Le Pelley \& McLaren, 2003). The source of the discrepancy remains to be resolved (but see Le Pelley, 2004).

Operant reinforcement. The third possibility also concerns changes in attention, but the mechanism involved is quite different from that just described and will operate even in the absence of explicit feedback (i.e., it could operate when the different stimuli are not explicitly followed by different consequences). Human subjects presented with a series of similar stimuli will attempt to detect differences among them. In some experiments, they are instructed to look for these differences, but they will undoubtedly do so even without instruction. And detecting one will probably give rise to a (mild) feeling of satisfaction. This self-generated event cannot operate in the same way as externally applied feedback does; it is the result of detecting a difference, not the cause of it. But, given that it will have rewarding properties, it may well have further effects. The argument is most simply made for the case of overt observing responses. Consider a complex visual display in which a distinctive feature is located in one corner. The reward of spotting this feature will reinforce the tendency to look toward that corner, and future discriminative performance will be enhanced. This attentional response, which is supported by operant conditioning (it is reinforced by its consequences), deserves, nonetheless, to be regarded as a mechanism of perceptual learning by our definition, since it serves to enhance the effectiveness of distinctive stimulus features. Although the example I have given concerns overt orienting to aspects of visual displays, the same principle could apply to 
Table 2

Experimental Designs

\begin{tabular}{clcl}
\hline Group & Preexposure & Conditioning & Test \\
\hline Experiment 1 & AX/BX_CX & AX+ & BX and CX \\
Experiment 2 & X/BX_CX & X+ & BX and CX \\
Experiment 3 & X/BX_CX & - & $\mathrm{B}+$ or C + \\
\hline
\end{tabular}

Note-A, B, and C represent different flavors that could be presented in compound with flavor $\mathrm{X}$. In preexposure, $\mathrm{AX}$ (or $\mathrm{X}$ ) was presented in alternation with BX. The CX compound was presented on a separate block of trials. In the conditioning phase of Experiments 1 and 2 and in the test phase of Experiment 3, flavors were followed by an injection of lithium chloride $(+)$

the reinforcement of covert attentional responses to other types of stimuli. It is difficult to imagine that an equivalent process could operate in nonhuman subjects that are given mere exposure to a pair of similar stimuli, and this difficulty gives a special importance to studies of such procedures. These will be considered next.

\section{Analyzing the Effects of Mere Exposure}

In recent years, versions of the rat flavor-aversion learning procedure presented in Table 1 have been used extensively to investigate the effects of exposure to stimuli on subsequent discrimination between them. Table 2 summarizes the designs of three such experiments. That labeled Experiment 1 provides a within-subjects demonstration of the effect previously reported by Symonds and Hall (1995). In this study (Blair \& Hall, 2003), the rats were preexposed to three different flavors (A, B, and C) that had been rendered more similar by the addition of a common flavor (X) to each of them. All of the rats received preexposure to $\mathrm{AX}$ and $\mathrm{BX}$, which were presented on alternate trials, and to CX, which was presented as a separate block of trials. They then received aversion conditioning with AX as the conditioned stimulus, followed by generalization tests with both BX and CX. The results of these tests (the amounts consumed of each compound flavor) are shown in the left panel of Figure 5. The aversion generalized to CX (consumption was suppressed), but the rats were more willing to consume BX; thus, alternating preexposure to $\mathrm{AX}$ and BX appeared to enhance the ability of the rats to discriminate between these two flavors.

The critical factor, therefore, in producing this version of the perceptual learning effect is that the stimuli to be discriminated on the test should be presented in alternation during preexposure - an outcome that accords with Gibson's (1969) view that perceptual learning will be facilitated when the subject is given the opportunity to compare the relevant stimuli. It certainly accords with the intuition that presenting similar stimuli in alternation will enable their distinctive features to stand out in some way - that presenting subjects with BX shortly after they have experienced $\mathrm{AX}$ (or vice versa) will allow them to notice $\mathrm{A}$ and $\mathrm{B}$ as being features that can occur independently of the common background, X. Hall (2003) attempted to develop and formalize this suggestion with his proposal that the experience of stimuli presented in alternation will enhance the effective salience of their distinctive features. The notion that experience can produce changes in the effective salience of a stimulus is not in itself controversial. The phenomenon of habituation (in which, with repeated presentation, a given stimulus starts to function as if its intensity had been reduced) is readily interpreted as reflecting a loss of effective salience. For the preexposure procedure used in Experiment 1 of Table 2, both the common $\mathrm{X}$ flavor and the control stimulus $\mathrm{C}$ can be expected to undergo such a loss during the repeated presentations of the CX compound; X would also suffer loss during the trials with AX and BX. Hall's (2003) suggestion was that the habituation effect can be reversed in the particular conditions of intermixed exposure, in which a compound containing a distinctive feature (such as BX) is alternated with the presentation of the background without the feature (such as that which occurs on an AX trial). Put informally, the suggestion was that the experience of $\mathrm{BX}$ will engender the expectation that $\mathrm{X}$ and $\mathrm{B}$ co-occur; the absence of B on the next AX trial would therefore be surprising, and the surprising omission of an event is assumed to boost its effective salience. (For a more formal account of this hypothesis, see Hall, 2003; Hall, Blair, \& Artigas, 2006.)

The proposal that the effective salience of Feature $\mathrm{C}$ is low, whereas that of Feature B is high, readily explains the results of the test phase of Experiment 1 (Figure 5). Conditioning with the AX compound will establish an aversion to $\mathrm{X}$ and thus produce generalization to the test stimuli, both of which contain the $\mathrm{X}$ element. But the ability of $\mathrm{X}$ to evoke its conditioned response (CR) will be modulated by the other stimuli that are present on the test. The salient B element will be more likely to interfere with the CR to X than will the less salient $C$ element, so that generalized responding will be less vigorous to $\mathrm{BX}$ than to $\mathrm{CX}$ - the result obtained. The remaining experiments summarized

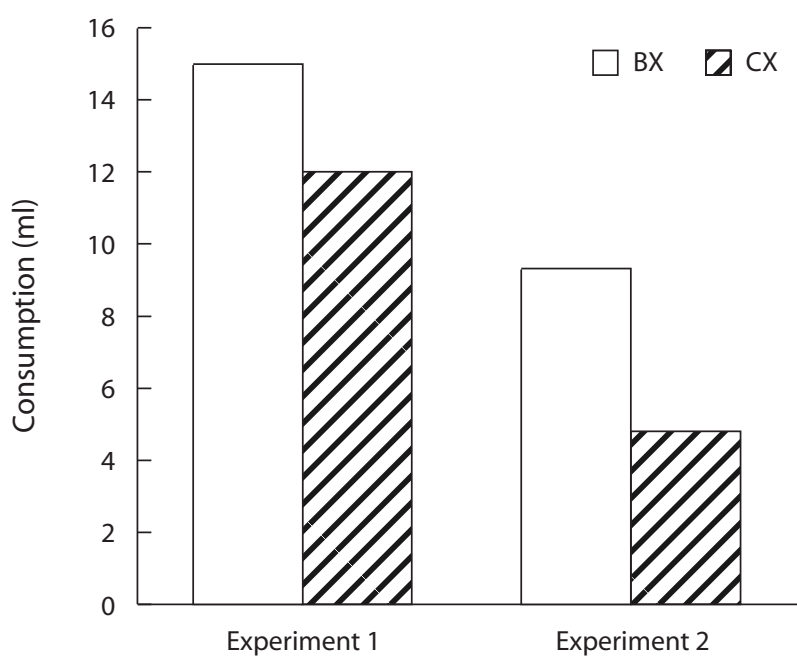

Figure 5. Results of the test phase (group mean consumption) for Experiments 1 and 2 of Table 2. After preexposure and conditioning, as was summarized in the table, all subjects received generalization tests with the compound flavors CX and BX. Experiment 1 was reported by Blair and Hall (2003); Experiment 2 by Hall, Blair, and Artigas (2006). 


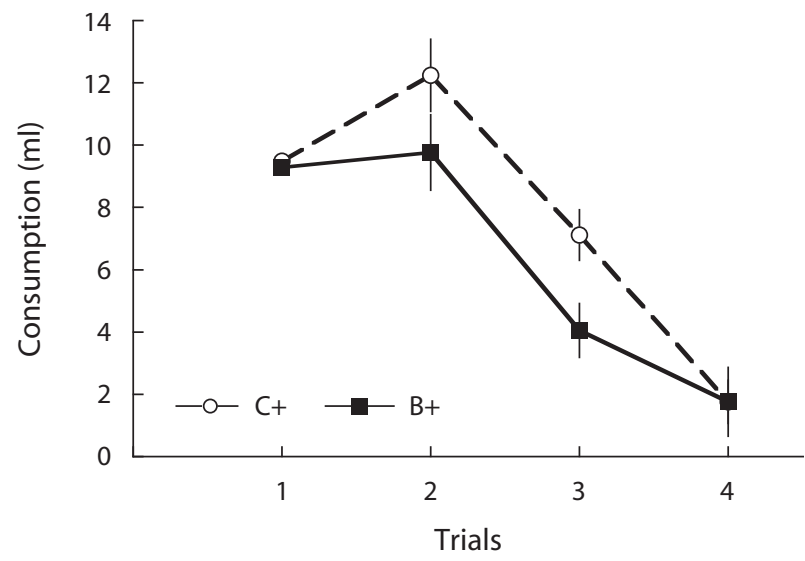

Figure 6. Results of the test phase (group mean consumption over reinforced trials) for Experiment 3 of Table 2. After the preexposure summarized in the table, one group of rats $(\mathrm{C}+)$ received a conditioning trial with Flavor $\mathrm{C}$ as the conditioned stimulus $(\mathrm{CS})$; a second group $(\mathrm{B}+)$ received Flavor $\mathrm{B}$ as the $\mathrm{CS}$. Experiment 3 was reported by Hall and Rodriguez (in press).

in Table 2 test further implications of this analysis of the basic perceptual learning effect.

In the study summarized as Experiment 2 in Table 2 (reported in full by Hall et al., 2006), the procedure was identical to that just described, except that the unique $\mathrm{A}$ feature was not used; preexposure consisted of alternating trials of $\mathrm{X}$ and $\mathrm{BX}$, and a block of $\mathrm{CX}$ trials. If what counts is the omission of an expected feature, then the alternation of X and BX (rather than of AX and BX) should be perfectly effective in enhancing the properties of $B$. The results (presented in Figure 5) show just this. Levels of consumption were lower than those for Experiment 1, which is to be expected since conditioning was given with $\mathrm{X}$ alone as the conditioned stimulus (CS), whereas the AX compound was used as the CS in Experiment 1. But, again, $\mathrm{BX}$ was consumed more readily than was $\mathrm{CX}$ - the result we have interpreted as indicating that the $C R$ to $X$ is better expressed in the presence of the less salient $C$ than in the presence of the more salient $B$.

If a difference in effective salience is indeed what characterizes the difference between Stimuli B and C after preexposure of the sort given in Experiment 2, this should be evident in tests other than the generalization test routinely used to assess discrimination in experiments on perceptual learning in animals. In Experiment 3 of Table 2, therefore, we turned to a much more direct assessment of the salience of these cues. In this study (reported by Hall \& Rodriguez, in press), rats were given preexposure to alternating presentations of $\mathrm{BX}$ and $\mathrm{X}$ and to a block of $\mathrm{CX}$ trials, just as in Experiment 2. For the test, they were divided into two groups: One received flavor-aversion conditioning with $\mathrm{B}$ as the CS, and one with $\mathrm{C}$ as the CS. The acquisition of a $\mathrm{CR}$ must be predicted to occur more quickly for a salient stimulus than for a less salient stimulus; that is, B is expected to condition more readily than $\mathrm{C}$. The acquisition of the aversion over the course of four conditioning trials is shown in Figure 6. On Trial 1, all subjects were given a fixed amount $(10 \mathrm{ml})$ of the test fluid, but thereafter, when free-access trials were given, the group that had been trained with B showed greater suppression of consumption than did the group that had been trained with $\mathrm{C}$.

\section{Extension to Mere Exposure Effects in Humans}

The designs and procedures employed in the experiments just described are very different from those used in studies of perceptual learning in people, but the experiments were conducted in the hope that they would help reveal learning mechanisms of general relevance. Empirical study will determine whether this hope is justified. Finally, I will report some recent experiments that have attempted to determine whether perceptual learning in humans displays the same features as those revealed by experiments on animals.

In order to carry out analytic studies, it is necessary for one to devise appropriate stimuli. These need to be difficult to discriminate - at least initially — and they should have identifiable unique and common features that can be manipulated independently. Figure 7 shows examples of checkerboard stimuli of the type used in a recent experiment by Lavis and Mitchell (2006; see also Mitchell, Kadib, Nash, Lavis, \& Hall, 2008; Mitchell, Nash, \& Hall, 2008) that meet these requirements. All have the same background pattern (constituting the common component, $X$ ) shown at the bottom of the figure. Each of the other four checkerboards shown in the figure has a unique feature added. In sum, these generate compounds to be referred to as $\mathrm{AX}, \mathrm{BX}, \mathrm{CX}$, and DX. In the figure, the unique features are shown outlined in black; this outline is for illustrative purposes and was not present on experimental trials. When given brief presentations of two such stimuli presented successively, people are initially unable to detect the difference between them. Can this be remedied by experience?

In their Experiment 1, Lavis and Mitchell (2006) carried out a study essentially equivalent to that done with flavor-aversion learning in rats, and it is presented as Experiment 1 of Table 2 . In pretraining, their subjects saw 60 presentations of each of four checkerboard stimuli. Each was presented for $700 \mathrm{msec}$, with an interval between trials of $700 \mathrm{msec}$. AX and BX were presented on alternating trials (the intermixed schedule); in the blocked phase, presentations of CX were given consecutively, followed by a block of presentations of DX. (The order of these phases was counterbalanced across subjects, as was the particular version of the checkerboard that was designated as $\mathrm{AX}, \mathrm{BX}, \mathrm{CX}$, or DX.) In the test phase, the subjects were confronted with a task in which the stimuli were associated with different outcomes - a procedure that parallels the generalization test procedure used with rats. Specifically, the subjects were required to learn a categorization task - to make one keypress to Stimulus AX and a different keypress to BX. Similarly, they had to learn to make different responses to CX and DX. Feedback was given. It was found that the discrimination between $\mathrm{AX}$ and $\mathrm{BX}$ was learned more readily than that between CX and DX. Over the course of testing with 30 presentations of each stimulus, the score for the AX/BX pair was over $84.1 \%$ correct; that for the CX/DX pair was $57.4 \%$. As was the 

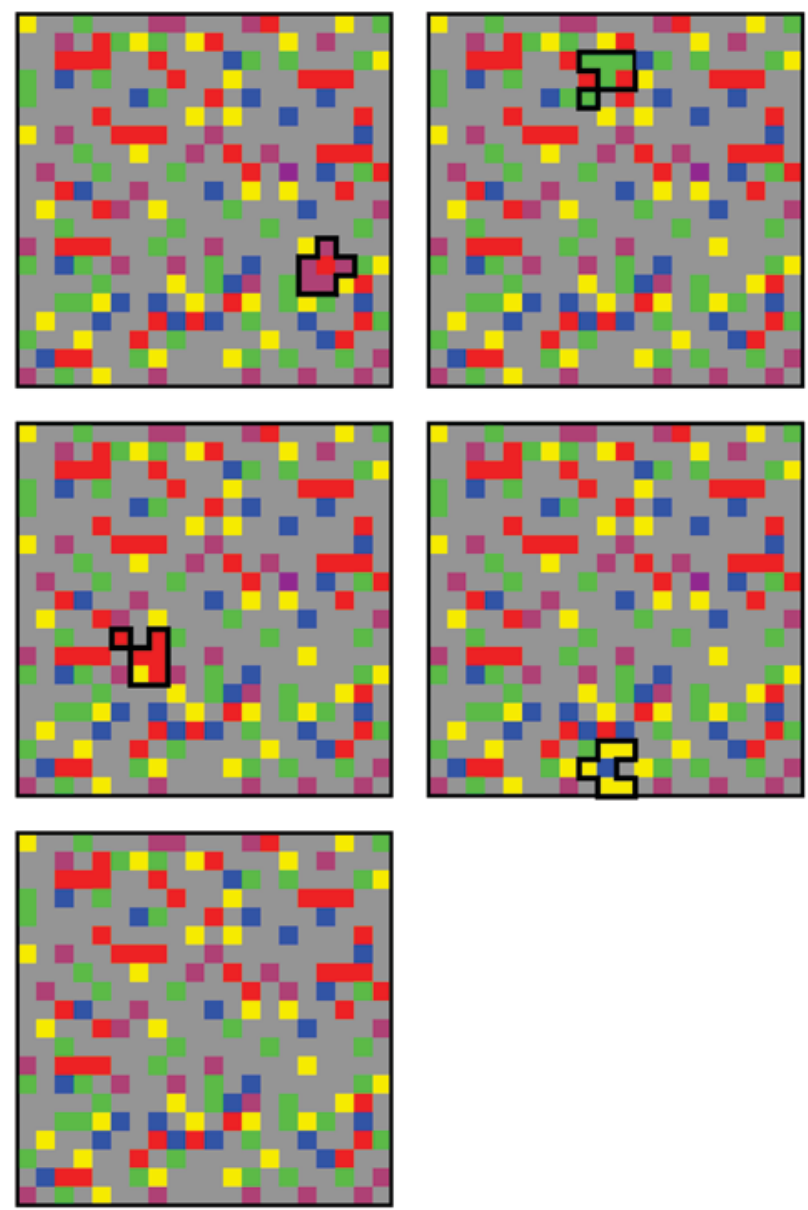

Figure 7. The stimuli used in the experiment by Mitchell, Nash, and Hall (2008). The display at the bottom is the common background $(X)$ used for all stimuli. In the other four, a distinctive feature has been added (a pattern of six adjacent colored squares). These features are outlined in black for the purpose of illustration. From "The Intermixed-Blocked Effect in Human Perceptual Learning Is Not the Consequence of Trial Spacing," by C. Mitchell, S. Nash, and G. Hall, Journal of Experimental Psychology: Learning, Memory, \& Cognition, 34, p. 238. Copyright 2008 by the American Psychological Association. Reprinted with permission.

case for rats, intermixed preexposure to similar stimuli was found to enhance subsequent discrimination between these stimuli.

In experiments with animal subjects, it is necessary for one to conduct explicit discrimination training (or a generalization test) to assess the effects of preexposure to the stimuli. The experiment just described adopted this sort of test. With human subjects, however, instruction can take the place of such training; that is, we can simply instruct them to report whether or not they can detect a difference between stimuli. In a further experiment, Lavis and Mitchell (2006) took this course. In their experiment, the preexposure procedure was identical to that just described. On the test trials, the subjects saw the stimuli presented in pairs (one for 3,500 msec, with a 550msec gap, and then the second, which stayed on until a response was made). Their task was to say "same" or "dif- ferent." There were four types of trials: those on which the stimuli-AX and BX - that had been intermixed during preexposure were presented (intermixed different trials); those in which $\mathrm{AX}$ and $\mathrm{AX}$ (or BX and BX) were presented (intermixed same); those in which CX and DX were presented (blocked different); and those in which CX and CX (or DX and DX) were presented (blocked same). The results (accuracy of performance on these trials) are presented in Figure 8. It is evident that accuracy in responding "same" when identical stimuli were presented was high, making it impossible to detect any difference between the intermixed and the blocked conditions, for both the intermixed and blocked stimuli. A difference was evident, however, on the different trials. The subjects continued to respond "same" to these (admittedly rather similar) blocked stimuli, but responded "different" to those given intermixed preexposure. Discrimination as assessed by the same-different test was better after intermixed than after blocked preexposure - an outcome readily explained by the proposal that perceptual effectiveness of unique features of the stimuli is higher after intermixed than after blocked preexposure.

\section{Conclusions}

This last experiment does something to bridge the gap between studies on perceptual learning in humans and in animals. The training procedures are directly derived from studies of the phenomenon in animals; the test procedure will be familiar to those who come to the subject from a background in human visual perception. But these are perhaps superficial matters; more solid common ground comes from a consideration of the theoretical analysis applied to both this and the other experiments described above.

Previous studies of perceptual learning in humans have used a range of varied procedures, and these seem to have given rise to a set of specialized mini-theories devised

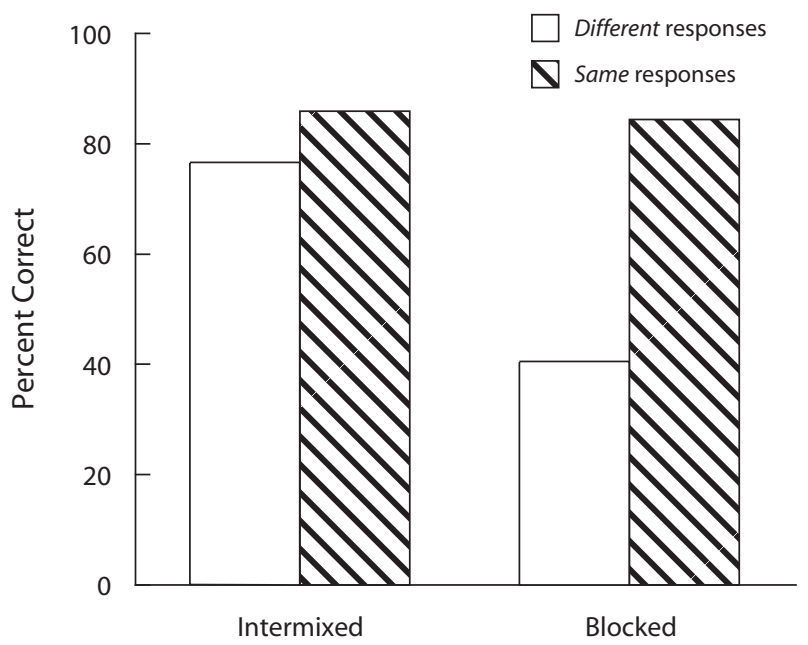

Figure 8. Results of the same-different test in the experiment by Lavis and Mitchell (2006). All subjects had received preexposure to a pair of checkerboards presented according to the intermixed schedule and to another pair presented according to a blocked schedule. 
solely to explain the effects seen in a particular experimental paradigm (see Hall, 2008). But what all these studies have in common (and share with those reported here, with both humans and animals) is that the procedure involves exposing the subjects to similar stimuli that have a set of common features (the $\mathrm{X}$ elements) and individual distinctive features (such as the A and B elements). When discrimination is improved after such experience-when the wine taster becomes able to distinguish claret from burgundy, the chicken-sexer between cocks and hens, or the experimental subject between one checkerboard and another-this is because their behavior has come to be controlled by the unique rather than the common features of the stimuli. Experimental studies designed to determine the nature of the learning mechanisms that generate such changes in the effectiveness of the component of complex stimuli will help us to understand perceptual learning generally, regardless of whether these studies are carried out with people in the visual psychophysics laboratory or with rats in the conditioning laboratory.

\section{AUTHOR NOTE}

This research was supported by grants from the United Kingdom Biotechnology and Biological Sciences Research Council, and the Australian Research Council. I thank Y. Lavis for helpful discussion. Correspondence concerning this article should be addressed to G. Hall, Department of Psychology, University of York, York YO10 5D, England (e-mail: g.hall@psych.york.ac.uk).

\section{REFERENCES}

BALl, K., \& SeKUler, R. (1982). A specific and enduring improvement in visual motion discrimination. Science, 218, 697-698.

Bennett, C. H., \& Mackintosh, N. J. (1999). Comparison and contrast as a mechanism of perceptual learning? Quarterly Journal of Experimental Psychology, 52B, 253-272.

Biederman, I., \& Shiffrar, M. M. (1987). Sexing day-old chicks: A case study and expert systems analysis of a difficult perceptuallearning task. Journal of Experimental Psychology: Learning, Memory, \& Cognition, 13, 640-645.

Blair, C. A. J., \& Hall, G. (2003). Perceptual learning in flavor aversion: Evidence for learned changes in stimulus effectiveness. Journal of Experimental Psychology: Animal Behavior Processes, 29, 39-48.

Bonardi, C., Graham, S., Hall, G., \& Mitchell, C. (2005). Acquired distinctiveness and equivalence in human discrimination learning: Evidence for an attentional process. Psychonomic Bulletin \& Review, 12, 88-92.

Demany, L. (1985). Perceptual learning in frequency discrimination. Journal of the Acoustical Society of America, 78, 1118-1120.

FAhle, M., \& Poggio, T. (2002). Perceptual learning. Cambridge, MA: MIT Press.

Fiorentini, A., \& Berardi, N. (1980). Perceptual learning specific for orientation and spatial frequency. Nature, 287, 43-44.

Gibson, E. J. (1969). Perceptual learning and development. New York: Appleton-Century-Crofts.

Hall, G. (1991). Perceptual and associative learning. Oxford: Oxford University Press, Clarendon Press.

HALL, G. (2003). Learned changes in the sensitivity of stimulus representations: Associative and nonassociative mechanisms. Quarterly Journal of Experimental Psychology, 56B, 43-55.

Hall, G. (2008). Perceptual learning. In J. Byrne (Editor-in-Chief) \& $\mathrm{R}$. Menzel (Vol. Ed.), Learning and memory: A comprehensive reference. Vol. 1: Learning theory and behavior (pp. 103-121). Amsterdam: Elsevier.

Hall, G., Blair, C. A. J., \& Artigas, A. A. (2006). Associative activation of stimulus representations restores lost salience: Implications for perceptual learning. Journal of Experimental Psychology: Animal Behavior Processes, 32, 145-155.

Hall, G., Mitchell, C., Graham, S., \& Lavis, Y. (2003). Acquired equivalence and distinctiveness in human discrimination learning: Evidence for associative mediation. Journal of Experimental Psychology: General, 132, 266-276.

Hall, G., \& Pearce, J. M. (1979). Latent inhibition of a CS during CS-US pairings. Journal of Experimental Psychology: Animal Behavior Processes, 5, 31-42.

Hall, G., \& Rodriguez, G. (in press). Factors determining the effects of associative activation on habituation. Journal of Experimental Psychology: Animal Behavior Processes.

Honey, R. C., Bateson, P., \& Horn, G. (1994). The role of stimulus comparison in perceptual learning: An investigation with the domestic chick. Quarterly Journal of Experimental Psychology, 47B, 83-103.

JAMES, W. (1890). The principles of psychology. New York: Holt.

Karni, A., \& SAGI, D. (1991). Where practice makes perfect in texture discrimination: Evidence for primary visual cortex plasticity. Proceedings of the National Academy of Sciences, 88, 4966-4970.

Lavis, Y., \& Mitchell, C. (2006). Effects of preexposure on stimulus discrimination: An investigation of the mechanisms responsible for human perceptual learning. Quarterly Journal of Experimental Psychology, 59, 2083-2101.

Le Pelley, M. E. (2004). The role of associative history in associative learning: A selective review and a hybrid model. Quarterly Journal of Experimental Psychology, 57B, 193-243

Le Pelley, M. E., \& Mclaren, I. P. L. (2003). Learned associability and associative change in human causal learning. Quarterly Journal of Experimental Psychology, 56B, 68-79.

MACKINTOSH, N. J. (1975). A theory of attention: Variations in the associability of stimuli with reinforcement. Psychological Review, 82, 276-298.

Mitchell, C., Kadib, R., Nash, S., Lavis, Y., \& Hall, G. (2008). Analysis of the role of associative inhibition in perceptual learning by means of the same-different task. Journal of Experimental Psychology: Animal Behavior Processes, 34, 475-485.

Mitchell, C., NASh, S., \& Hall, G. (2008). The intermixed-blocked effect in human perceptual learning is not the consequence of trial spacing. Journal of Experimental Psychology: Learning, Memory, \& Cognition, 34, 237-242.

Mondragón, E., \& Hall, G. (2002). Analysis of the perceptual learning effect in flavour aversion learning: Evidence for stimulus differentiation. Quarterly Journal of Experimental Psychology, 55B, 153-169.

Mundy, M. E., Dwyer, D. M., \& Honey, R. C. (2006). Inhibitory associations contribute to perceptual learning in humans. Journal of Experimental Psychology: Animal Behavior Processes, 32, 178-184.

Mundy, M. E., Honey, R. C., \& Dwyer, D. M. (2007). Simultaneous presentation of similar stimuli produces perceptual learning in human picture processing. Journal of Experimental Psychology: Animal Behavior Processes, 33, 124-138.

Myles-Worsley, M., Johnston, W. A., \& Simons, M. A. (1988). The influence of expertise on X-ray image processing. Journal of Experimental Psychology: Learning, Memory, \& Cognition, 14, 553-557.

Pearce, J. M., \& Hall, G. (1980). A model for Pavlovian learning: Variations in the effectiveness of conditioned but not of unconditioned stimuli. Psychological Review, 87, 532-552.

Rescorla, R. A., \& Wagner, A. R. (1972). A theory of Pavlovian conditioning: Variations in the effectiveness of reinforcement and nonreinforcement. In A. H. Black \& W. F. Prokasy (Eds.), Classical conditioning II: Current research and theory (pp. 64-99). New York: Appleton-Century-Crofts.

ShIU, L.-P., \& PASHLER, H. (1992). Improvement in line orientation discrimination is retinally local but dependent on cognitive set. Perception \& Psychophysics, 52, 582-588.

SyMONDS, M., \& HALL, G. (1995). Perceptual learning in flavor aversion conditioning: Roles of stimulus comparison and latent inhibition of common stimulus elements. Learning \& Motivation, 26, 203-219.

(Manuscript received November 17, 2008; accepted for publication February 18, 2009.) 